\title{
Results from CMS and ATLAS: Electroweak Symmetry, Breaking and Beyond
}

\author{
Paolo Azzurri*ł \\ CERN, European Organization for Nuclear Research, Geneva, Switzerland \\ INFN Sezione di Pisa, Pisa, Italy \\ E-mail: Paolo.Azzurri@cern.ch
}

\begin{abstract}
The discovery of the Higgs boson, announced by the CMS and ATLAS collaborations in 2012, unearthed the final cornerstone of the standard electroweak model of particle physics, and represents the main legacy of the LHC Run 1. With Run 1 data the mass of the Higgs boson has been determined with $0.2 \%$ precision, while coupling properties are only established at the $10 \%$ level or worse. As the picture of the minimal standard model is now complete, unsettled difficulties and open questions remain on its stage. The LHC Run 2 has successfully started in 2015, opening a new period of particle physics exploration, at higher energy and intensity: it will undoubtedly deliver more insight on the electroweak model, its symmetry breaking mechanism, and on possible solutions to its difficulties.
\end{abstract}

54th International Winter Meeting on Nuclear Physics 25-29 January 2016

Bormio, Italy

* Speaker.

${ }^{\dagger}$ on behalf of the CMS and ATLAS collaborations 


\section{Introduction}

The LHC collider at CERN successfully operated during Run 1 (2009-2012) delivering about $5 \mathrm{fb}^{-1}$ and $20 \mathrm{fb}^{-1}$ of proton-proton (pp) collisions respectively at $7 \mathrm{TeV}$ and $8 \mathrm{TeV}$ center of mass energies. The LHC Run 2 data taking period started in 2015, delivering about $3 \mathrm{fb}^{-1}$ of pp collisions at $13 \mathrm{TeV}$ center of mass energy.

In the following the CMS detector and performances with 2015 data will be summarised, and selected initial results with $13 \mathrm{TeV}$ data will be given, both from the CMS and ATLAS experiments, in the domains of electroweak standard model (SM) measurements, Higgs boson measurements, and searches for supersymmetry. Combined Run 1 results on the Higgs boson mass and properties determinations will also be discussed.

\section{The CMS Detector}

The CMS detector [1] is one of the two large multipurpose particle detectors operated at the LHC, the second multipurpose-detector, ATLAS [2], is described in more detail in S. Bethke's presentation [3] at this conference. The central feature of the CMS apparatus is a superconducting solenoid of $6 \mathrm{~m}$ internal diameter, providing a magnetic field of $3.8 \mathrm{~T}$. A silicon pixel and strip tracker, a lead tungstate crystal electromagnetic calorimeter (ECAL), and a brass and scintillator hadron calorimeter (HCAL) are located within the axial field. Muons are measured in gas-ionization detectors embedded in the steel flux-return yoke of the solenoid. Forward calorimetry (pseudorapidity $3<|\eta|<5$ ) complements the coverage provided by the barrel $(|\eta|<1.3$ ) and endcap $(1.3<|\eta|<3)$ detectors. The first level (L1) of the CMS trigger system, composed of specialized processors, uses information from the calorimeters and muon detectors to select the most interesting events in a time interval of less than $4 \mu \mathrm{s}$. The high-level trigger (HLT) processor farm decreases the event rate from about $100 \mathrm{kHz}$ to about $1 \mathrm{kHz}$, before data storage.
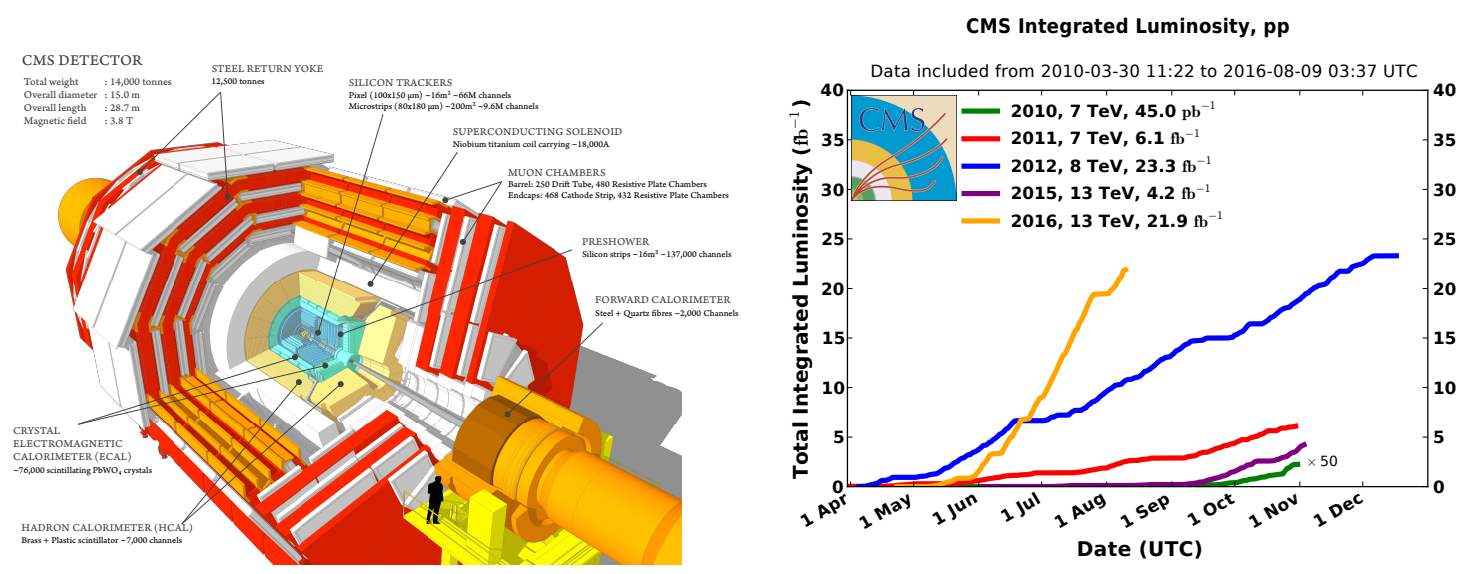

Figure 1: Sectional view of the CMS detector (left), and cumulative luminosity versus day delivered to CMS during stable beams for pp collision.

A sketch of the CMS detector is shown in Figure 1, together with a plot of delivered data luminosity during Run1 and Run 2 proton-proton (pp) collisions. The total pp integrated luminosity 
recorded by CMS in Run 1 amounts to approximately $5 \mathrm{fb}^{-1}$ at $7 \mathrm{TeV}$, and $20 \mathrm{fb}^{-1}$ at $8 \mathrm{TeV}$. During the 2015, the CMS data taking efficiency was above $90 \%$, recording a total integrated luminosity of about $3.8 \mathrm{fb}^{-1}$. Around one quarter of the data was recorded without magnetic field, due to problems in operating the liquid Helium cryogenic system.

\subsection{Event reconstruction and performances with 2015 data}

The CMS offline analysis uses reconstructed charged-particle tracks and candidates from the particle-flow (PF) algorithm $[4,5,6]$. In the PF event reconstruction all stable particles in the event, i.e. electrons, muons, photons, and charged and neutral hadrons, are reconstructed as PF candidates using information from all CMS subdetectors to obtain an optimal determination of their direction, energy, and type.

The PF candidates are then used to reconstruct the jets and missing transverse energy. A few plots showing the performances for the reconstruction of photons, electrons and muons with 2015 proton collision data at $13 \mathrm{TeV}[7,8]$ are shown in Fig. 2.

Jets are reconstructed by clustering PF candidates with the anti- $k_{T}$ algorithm $[9,10]$ with a distance parameter of 0.4 . Reconstructed jets require a small additional energy correction, mostly due to thresholds on reconstructed tracks and clusters in the PF algorithm and various reconstruction inefficiencies [11]. Jet identification criteria are also applied to reject misreconstructed jets resulting from detector noise, as well as jets heavily contaminated with pileup energy (clustering of energy deposits not associated with a parton from the primary pp interaction) [12]. The efficiency of the jet identification criteria is greater than $99 \%$, with the rejection of $90 \%$ of background pileup jets with $p_{\mathrm{T}} \simeq 50 \mathrm{GeV}$.

The identification of jets that originate from the hadronization of $b$ quarks is done with the CSV $\mathrm{b}$ tagger $[13,14]$. The CSV algorithm combines the information from track impact parameters and secondary vertices identified within a given jet, and provides a continuous discriminator output.

Figure 3 shows the performances for the CMS jet energy calibration [15], and for the signed impact parameters of tracks, relevant for b-tagging [16], with 2015 proton collision data at $13 \mathrm{TeV}$.

\section{Electroweak measurements}

\section{1 $\mathrm{W}$ and $\mathrm{Z}$ production}

Measurements of total and fiducial $\mathrm{W}$ and $\mathrm{Z}$ boson production cross sections in the electron and muon final states have been performed by CMS [17] and ATLAS [18] with the initial 2015 proton collision data at $13 \mathrm{TeV}$.

Figure 4 shows some important distributions for the inclusive $\mathrm{W}$ and $\mathrm{Z}$ boson analysis as the missing transverse energy $\left(E_{\mathrm{T}}^{\text {miss }}\right)$ and transverse mass for leptonic decays of the $\mathrm{W}$ boson, and the dilepton invariant mass and rapidity for the $\mathrm{Z}$ boson measurements.

The measured cross section values agree with the energy dependence predicted by next-tonext-to-leading-order (NNLO) calculations, as shown in Fig. 5. Fiducial measurements are also compared to predictions of different parton distribution functions (PDFs), shown in the bottom plots of Fig. 5. The ratio of rates in the electron and muon channels can also serve as interesting confirmations of the lepton universality of weak interactions, also shown in Fig. 5. 

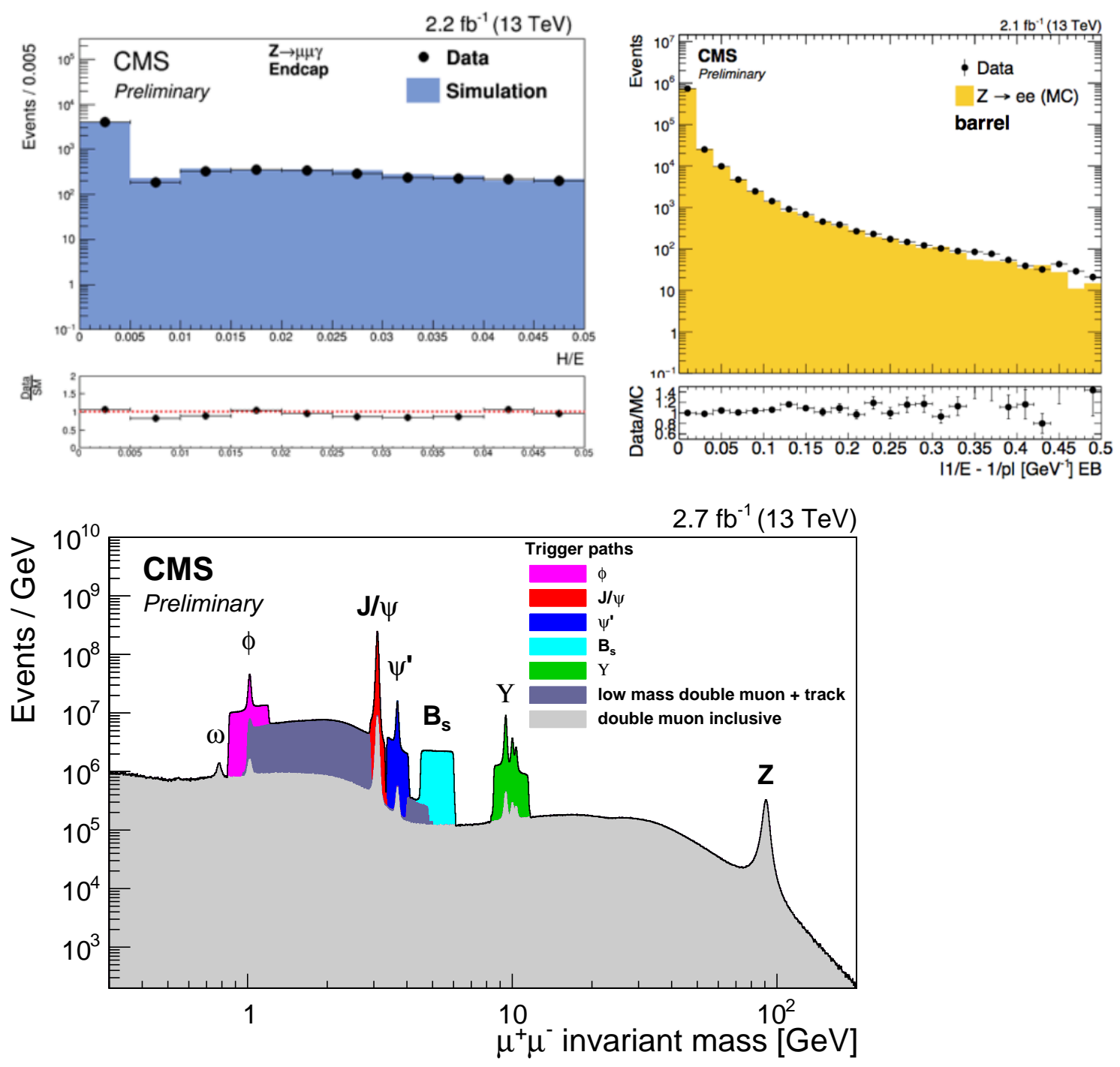

Figure 2: CMS event reconstruction performances with 2015 proton collision data at $13 \mathrm{TeV}$. Ratio of energy in the HCAL to the energy of the ECAL for selected photons in the ECAL barrel from $\mathrm{Z} \rightarrow \mu \mu \gamma$ events [7] (top left). Difference between the inverse ECAL energy and the inverse tracker momentum for $\mathrm{Z} \rightarrow$ ee electrons in the ECAL barrel [7] (top right). Dimuon mass distribution collected with various dimuon triggers [8] (bottom).

\subsection{Diboson production}

The production of electroweak boson pairs are very intriguing processes in the SM where large negative interference effects (related to the gauge theory cancellations) dominate production mechanisms, guaranteeing the tree level scattering unitarity, and the perturbative renormalizability. This scenario makes diboson productions extremely sensitive to the presence and possible anomalies in the structure of the SM gauge self-couplings, a salient feature of the electroweak model.

Final results with Run 1 data on W-pair productions have been published [19][20], and Fig. 6 shows the level of the contraints on the gauge self couplings. 

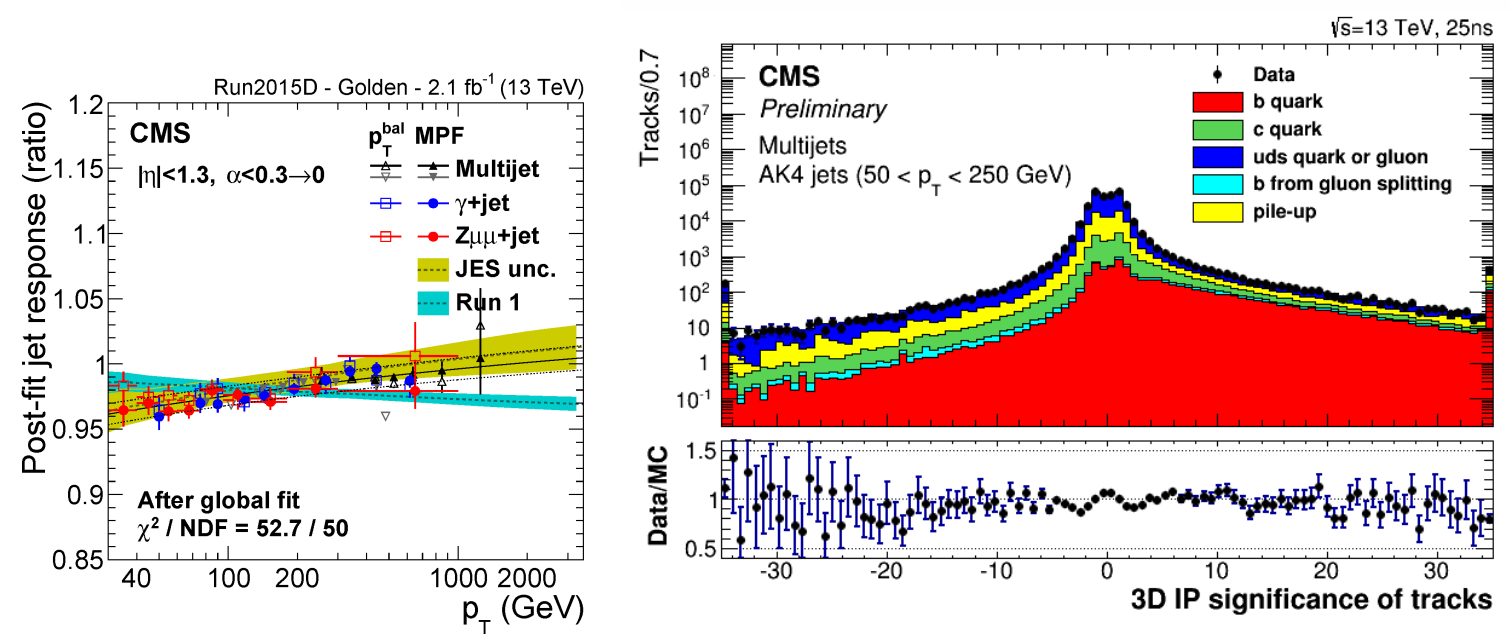

Figure 3: CMS event reconstruction performances with 2015 proton collision data at $13 \mathrm{TeV}$. Jet energy calibration with dijets, $\gamma+$ jet and $Z+$ jet events [15] (left). Impact parameter significance of tracks in jets for b-tagging [16] (right).

The WZ production is similar and complementary to the W-pair production. First results on WZ productions have been reported by CMS [21] with $1.34 \mathrm{fb}^{-1}$ of proton collision data at $13 \mathrm{TeV}$, in the leptonic decay modes $\mathrm{WZ} \rightarrow \ell v \ell^{\prime} \ell^{\prime}$, where $\ell, \ell^{\prime}=\mathrm{e} \mu$, and shown in Fig 7. The measured cross section $\sigma(\mathrm{pp} \rightarrow \mathrm{WZ})=36.8 \pm 4.6$ (stat) ${ }_{6.2}^{+8.1}$ (syst) \pm 0.6 (theo) \pm 1.7 (lumi) pb, for Z bosons produced in the mass region $60<m_{\mathrm{Z}}<120 \mathrm{GeV}$, is consistent with SM predictions.

The Z-pair production does not have negative gauge cancellation effects, as no gauge selfcouplings are predicted to contribute. Measuring the process is still sensitive to possible anomalies due to non standard neutral gauge couplings. The first Z-pair measurements with $13 \mathrm{TeV}$ pp collision data, shown in Figs. 8-9, have been released by CMS [23] and ATLAS [22] with electron and muon decay modes.

\section{The Higgs boson}

In the minimal standard model [24] [25] [26] the Brout-Englert-Higgs mechanism [27] [28] [29] explains the electroweak symmetry breaking and allows electroweak gauge bosons to acquire mass. The mechanism predicts the existence of a Higgs scalar field, that has been experimentally searched for since its proposal. Strong indirect hints for the existence of a Higgs boson came from the precision electroweak determinations before the start of the LHC, mostly from LEP data, indicating the presence of a Higgs boson in the 115-150 GeV mass range [30]. The subsequent observation in 2012 with the LHC Run 1 proton-proton collision data by both the CMS [31] and ATLAS [32] collaborations achieved one of the main goals of the LHC physics program.

\subsection{Higgs boson mass}

The Higgs boson mass $\left(m_{\mathrm{H}}\right)$ is the only free parameter of the Higgs sector in the minimal SM. The ATLAS and CMS Collaborations have independently measured $m_{\mathrm{H}}$ using Run 1 samples of 

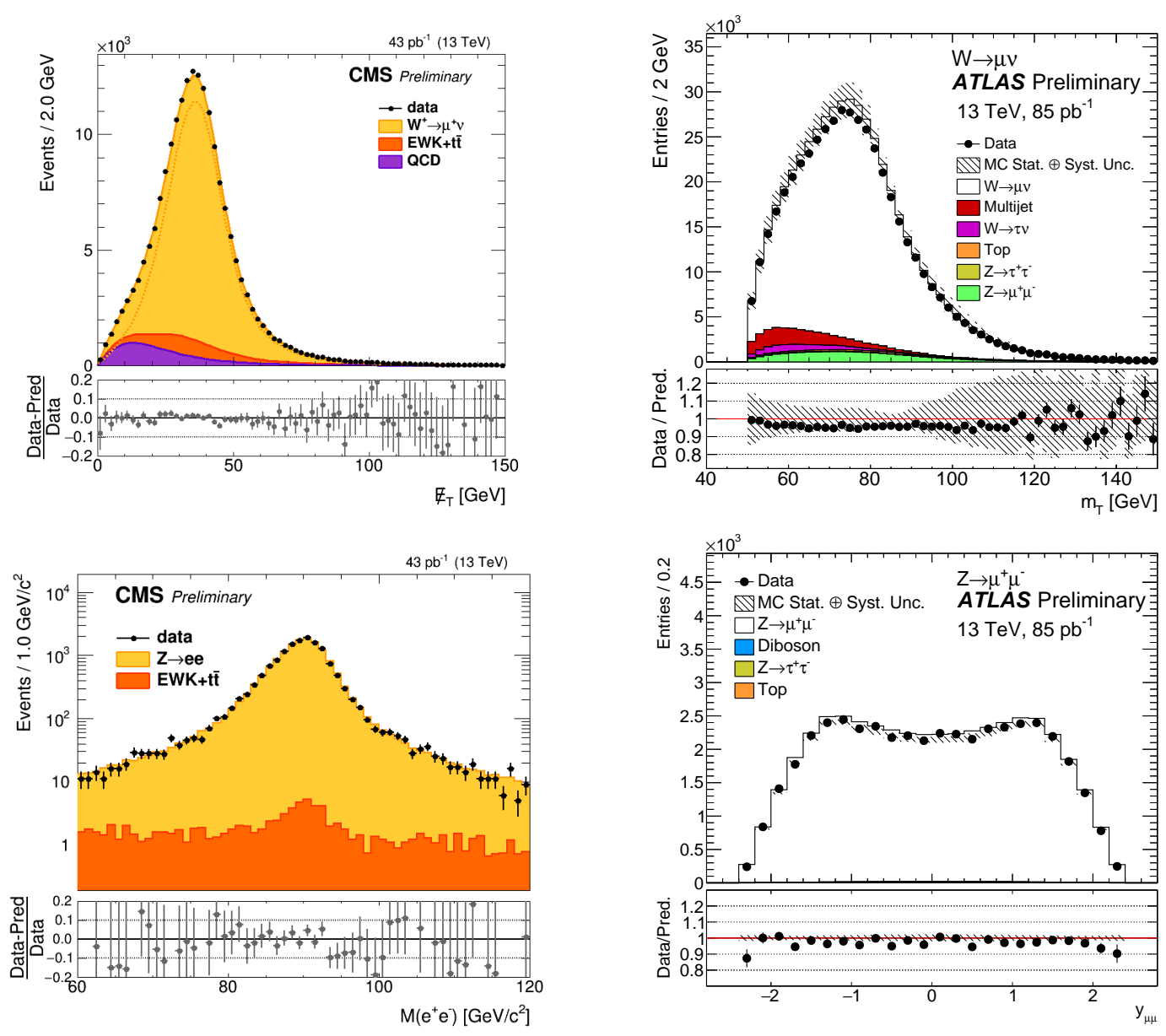

Figure 4: Inclusive $\mathrm{W}$ and $\mathrm{Z}$ boson productions with 2015 proton collision data at $13 \mathrm{TeV}$ [17, 18]. The CMS missing transverse energy distributions for $\mathrm{W}^{+}$boson candidate events in the muon final state (top left). Transverse mass distribution, calculated from the lepton and the $E_{\mathrm{T}}^{\text {miss }}{ }_{\text {vectors for selected } \mathrm{W} \rightarrow \mu \nu}$ ATLAS events (top right). The CMS dilepton mass distributions for $Z$ boson events in the electron final state (bottom left). $\mathrm{Z}$ boson rapidity distribution in the ATLAS $\mathrm{Z} \rightarrow \mu \mu$ channel (bottom right).

proton-proton collision using the mass peaks observed in the diphoton $(\gamma \gamma)$ and four-lepton $(4 \ell)$ decay channels, these results have been combined [33] yielding

$$
m_{\mathrm{H}}=125.09 \pm 0.21 \text { (stat) } \pm 0.11 \text { (syst) } \mathrm{GeV}
$$

where the total uncertainty is dominated by the statistical term, with the systematic uncertainty dominated by effects related to the photon, electron, and muon energy or momentum scales and resolutions.

\subsection{Higgs boson couplings}

Combined ATLAS and CMS measurements of the Higgs boson production and decay rates, and constraints on its couplings to vector bosons and fermions have been produced using Run 1 data [36]. 

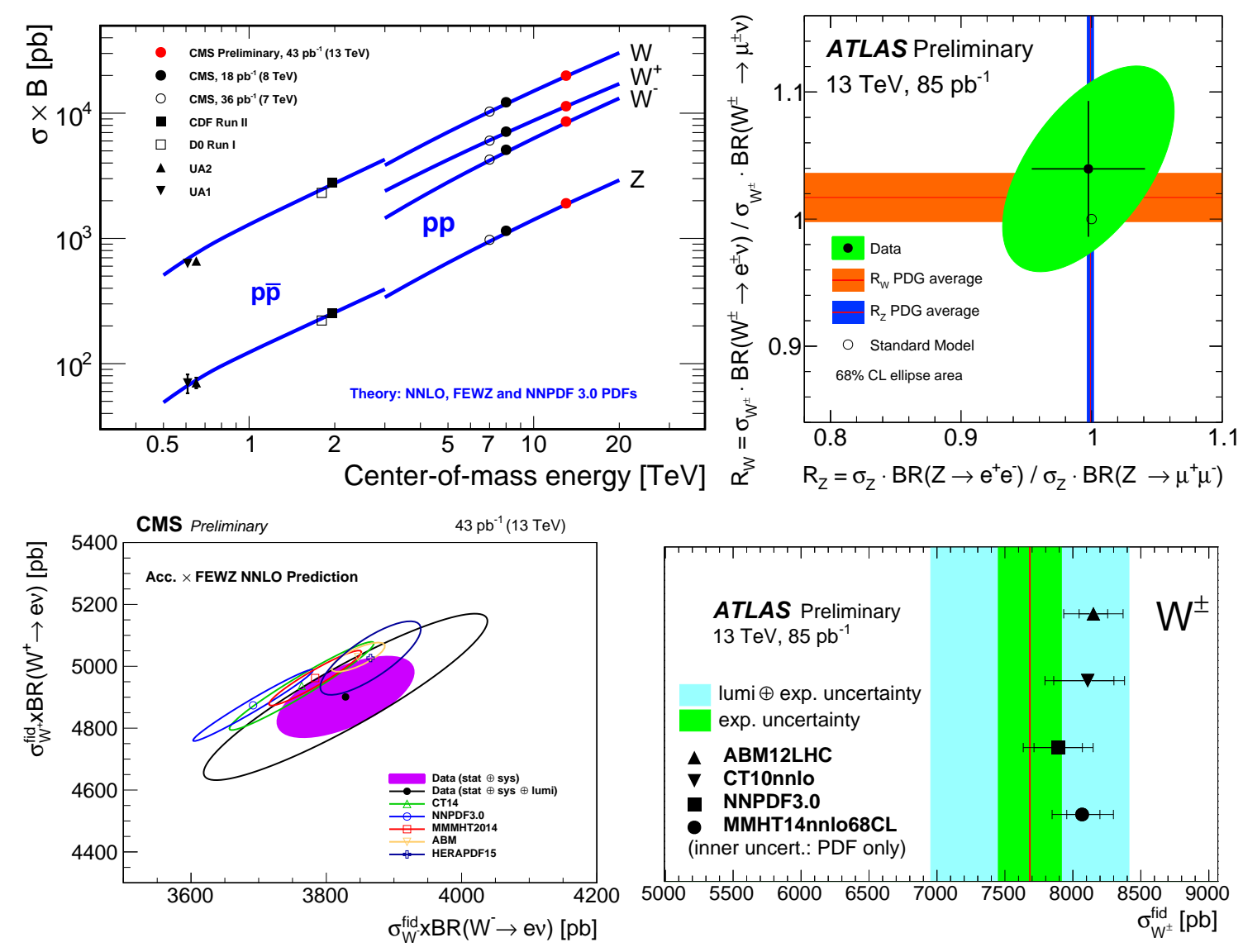

Figure 5: Measurements of the total $\mathrm{W}, \mathrm{W}^{+}, \mathrm{W}^{-}$and $\mathrm{Z}$ production cross sections versus center-of-mass energy for CMS and experiments at lower-energy colliders [17] (top left). Ratio of ATLAS Z and Wboson production cross sections in the electron and muon channels, compared to SM and other experimental checks of lepton universality [18] (top right). CMS measured and predicted $\mathrm{W}^{+}$versus $\mathrm{W}^{-}$fiducial inclusive production cross sections in the electron channel: the ellipses illustrate the data $68 \% \mathrm{CL}$ coverage and the theoretical predictions for five PDF sets [17] (bottom left). ATLAS measured and predicted Z fiducial cross section for four PDF sets [18] (bottom right).

In the SM, Higgs boson production at the LHC mainly occurs through the processes shown in Fig. 11, while the main searched decay modes are shown in Fig. 12. Decays to heavy bosons (W and $\mathrm{Z}$ ) and fermions (b-quarks) proceed through tree-level processes whereas the diphoton decay is mediated by $\mathrm{W}$-boson and heavy quark (b or t) loops.

The Higgs boson production and decay rates of the two experiments in all searched modes are combined with two generic parameterisations based on ratios of cross sections and branching ratios, and based on ratios of coupling modifiers. Measurements are performed using a combined likelihood of about 580 signal and control distributions. Care is taken in building the acceptance matrix and in treating correlations among over four thousand systematic effects.

Results show a general consistency of data with the Standard Model predictions for all parameterisations considered. The total combined Higgs boson signal yield relative to the Standard Model prediction is measured to be $\mu=1.09 \pm 0.11$ and is the most constrained and precise result 

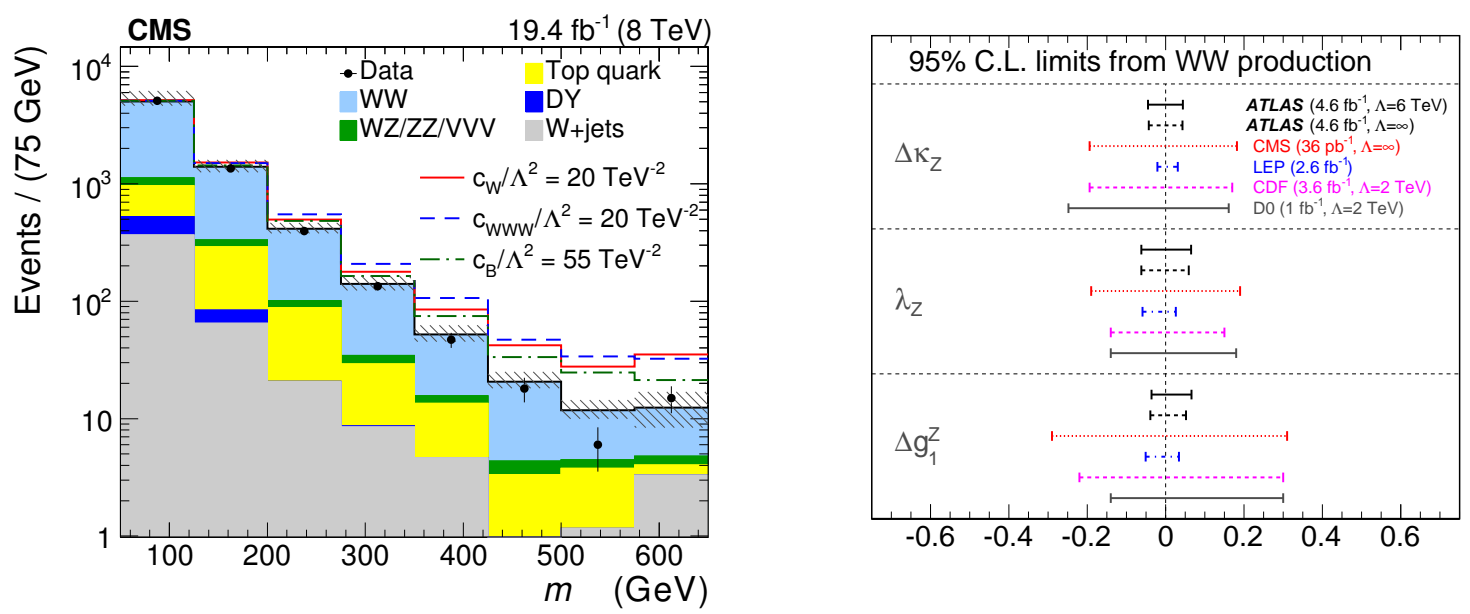

Figure 6: Measurements of W-pairs with Run 1 data. The $m_{\ell \ell}$ distribution with all SM backgrounds and anomalous gauge couplings associated with dimension-six operators [19] (left). Comparison of anomalous TGC limits from ATLAS, CMS, CDF, D0 and LEP experiment [20] (right).
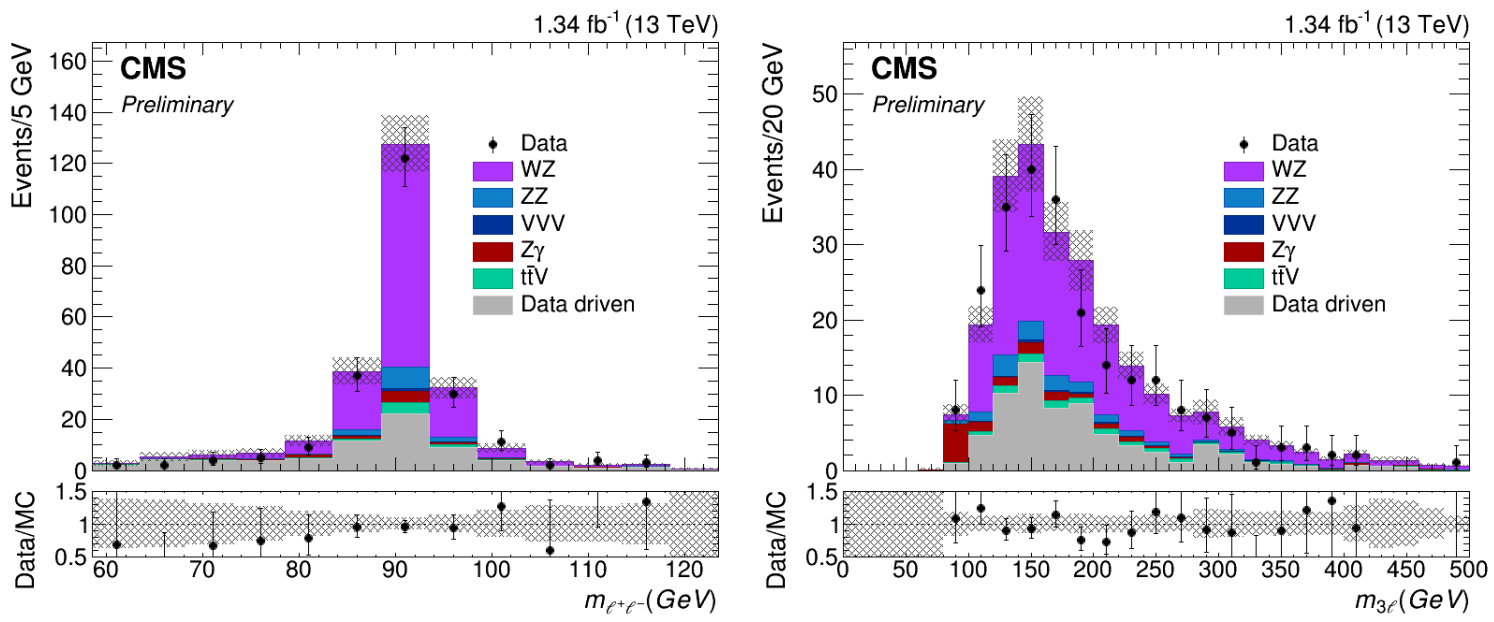

Figure 7: Measurements of WZ with initial Run 2 data [21]. Distribution of the $\mathrm{Z}$ reconstructed mass summed for all decay channels (left). Distribution of the trilepton reconstructed mass summed for all decay channels (right). Solid symbols represent the data with statistical uncertainty, shaded histograms represent the expected WZ signal and backgrounds.

obtainable from the data.

The results of fitting SM-relative signal strengths for independent productions or decay modes are shown in Fig. 13. The most remarkable discrepancy appears for the ditop-associated production, that is measured to be 2.3 standard deviations above the SM prediction. The combined observed significance for the vector boson fusion production process is 5.4 standard deviations, while the observed significance for the ditau decay is 5.5 standard deviations.

Results of the fits to Higgs boson couplings modifiers in the parameterisations assuming the SM structure are shown in Fig. 14 where the mass dependence is also shown. Results when fitting independently also the Higgs coupling to photons and gluons, focusing towards a BSM scenario, 

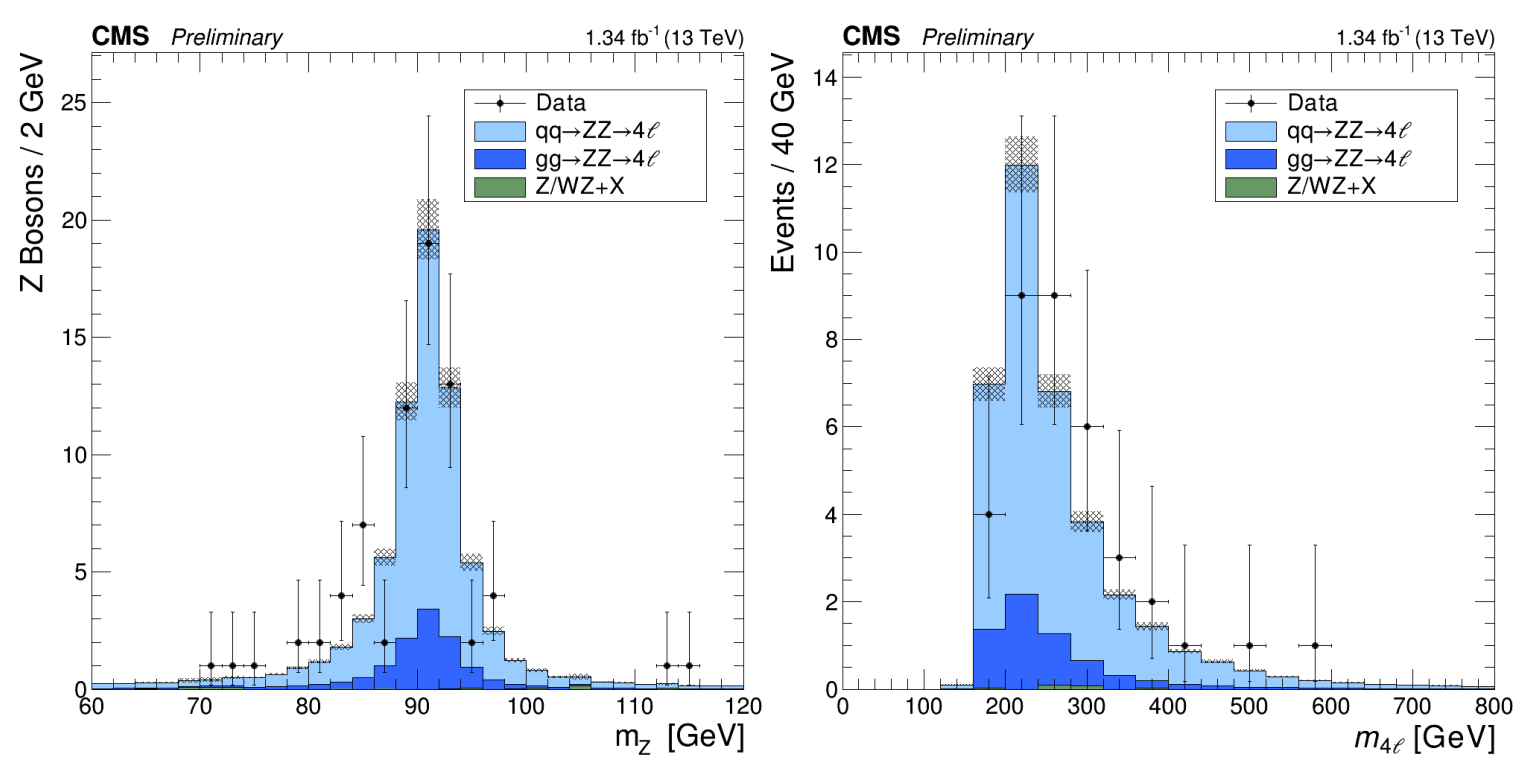

Figure 8: CMS measurements of Z-pairs with initial Run 2 data [23]. Distribution of the reconstructed mass of all $\mathrm{Z}$ bosons in $\mathrm{ZZ}$ events (left) and $m_{\mathrm{ZZ}}$ (left), summed over the $4 \mathrm{e}, 2 \mathrm{e} 2 \mu$, and $4 \mu$ final states. The results correspond to an integrated luminosity of $1.34 \mathrm{fb}^{-1}$. Points represent the data, shaded histograms represent the expected ZZ signal.
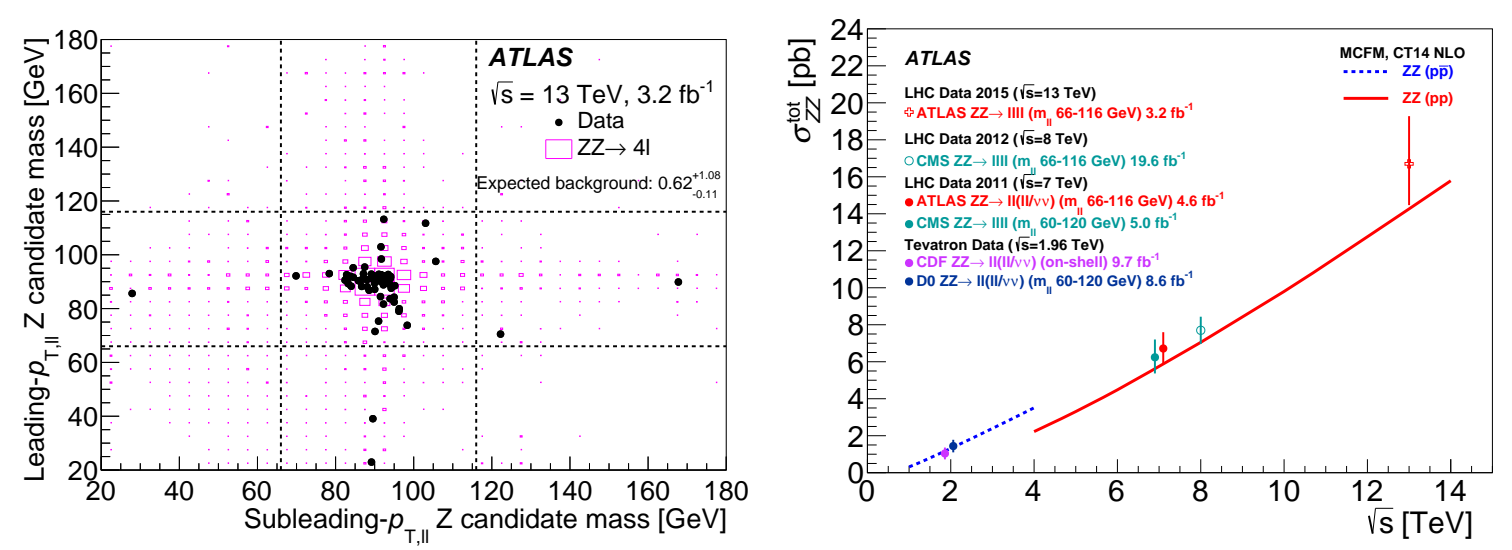

Figure 9: ATLAS measurements of Z-pairs with initial Run 2 data [22]. Invariant mass $m_{\ell \ell}$ of the leading$p_{\mathrm{T}}$, versus the subleading- $p_{\mathrm{T}}$, lepton pair $(\ell \ell)$ : points represent experimental data and filled histograms show the signal prediction from simulation (right). Total cross section compared to measurements at lower center-of-mass energies by ATLAS, CMS, CDF, and D0, and to a prediction from MCFM (left).

are shown in Fig. 15.

Finally results expressed in generic terms of ratios of production cross sections and of decay branching ratios, relative to gluon-fusion production and decays to Z-pairs, are shown in Fig. 16. This allows for a more generic exploration of deviations from SM predictions, with reduced systematic uncertainties. In this representation the relative deficit of decays to b-quarks, also present in other parameterisations, is enhanced by $\mathrm{VH}$ and $\mathrm{ttH}$ signal excesses in other decay modes.

Figure 16 also shows the log-likelihood profile of the bb/ZZ ratio, nomalised to SM predic- 

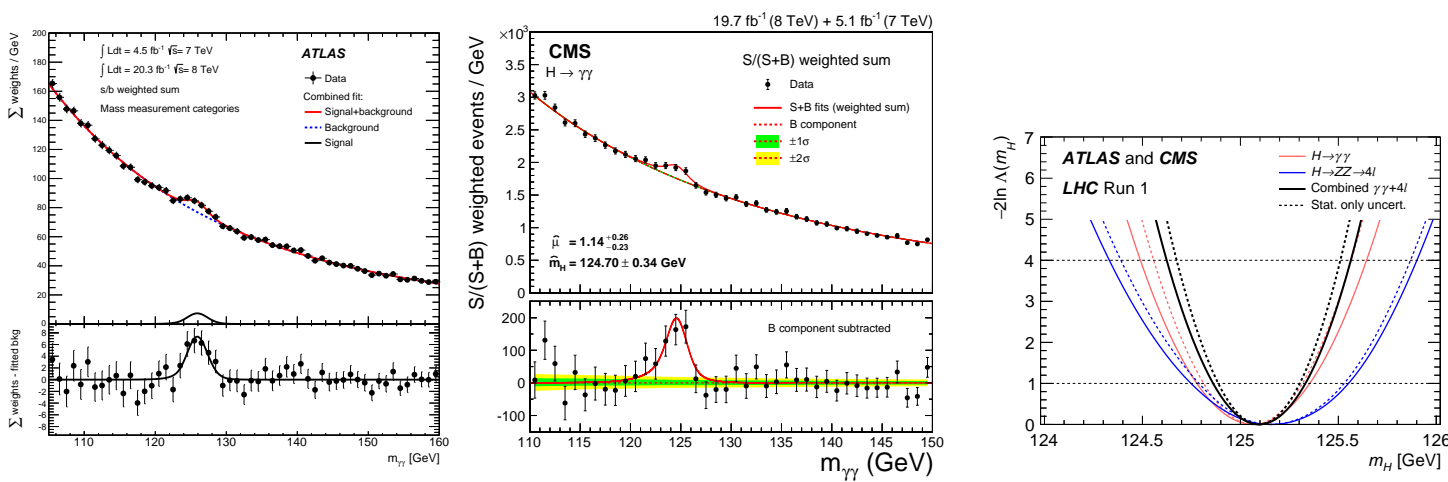

Figure 10: ATLAS diphoton mass distribution, showing weighted data points with errors, and the result of the fitted signal and background [34]. The bottom plot shows the difference between the summed weights and the background component of the fit (left). CMS diphoton mass spectrum weighted by the signal probability in each event class, together with the background subtracted weighted mass spectrum [35] (center). Scans of twice the negative log-likelihood ratio as functions of the Higgs boson mass $m_{\mathrm{H}}$ for the ATLAS and CMS combination of the diphoton (red), four-lepton (blue), and combined (black) channels [33]. The dashed curves show the results accounting for statistical uncertainties only. The 1 and 2 standard deviation limits are indicated by the intersections of the horizontal lines at 1 and 4, respectively, with the log-likelihood scan curves. (right).
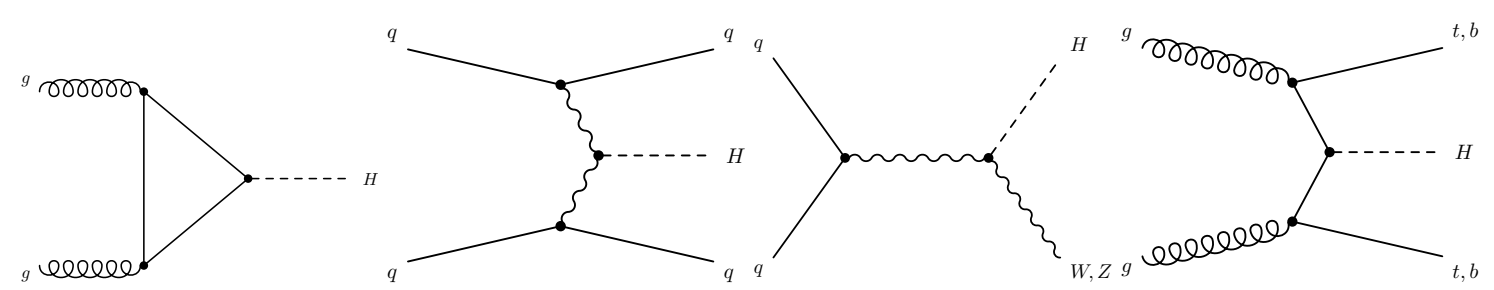

Figure 11: Diagrams for Higgs boson production at the LHC via the gluon fusion (ggF), vector boson fusion $(\mathrm{VBF})$, vector associated $(\mathrm{VH})$, and ditop associated $(\mathrm{ttH})$ production processes.

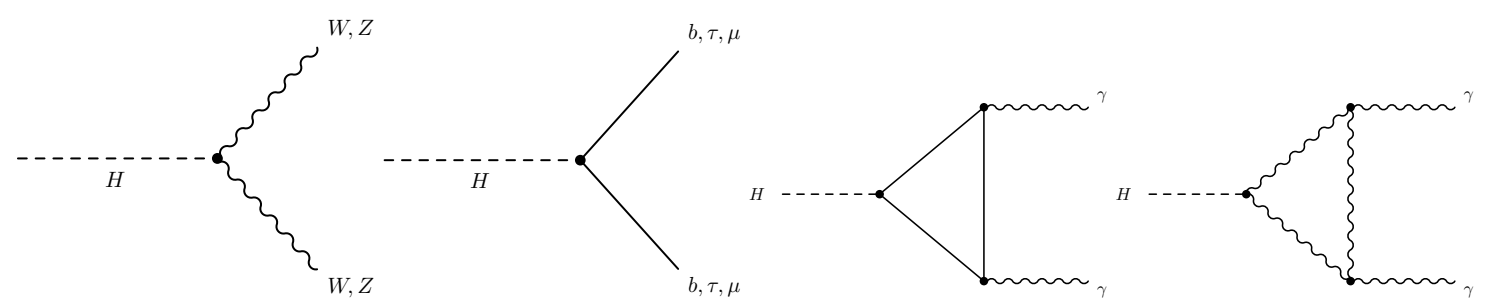

Figure 12: Diagrams for main Higgs boson decays relevant at the LHC: to boson pairs (far left), to fermion pairs (center left), to photon pairs via heavy fermion (center right) or heavy boson loops (far right). 

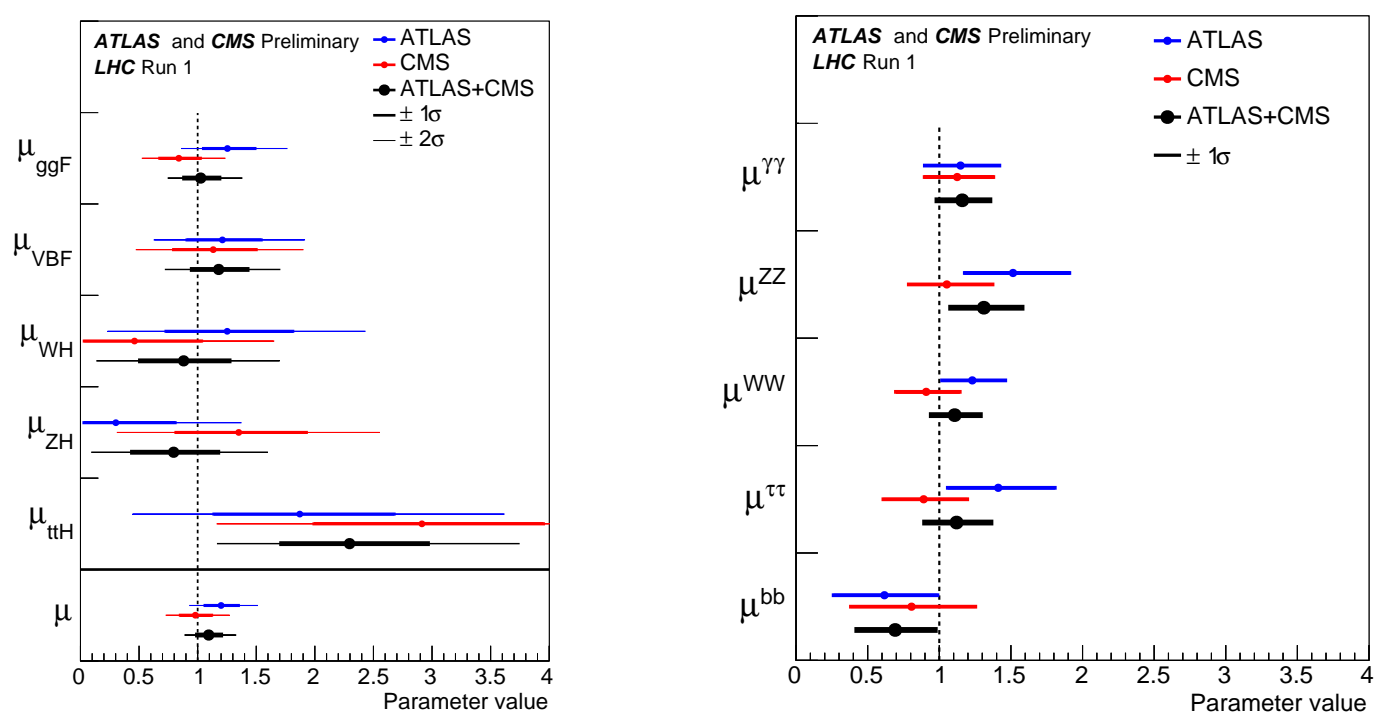

Figure 13: Results for Higgs boson production (left) and decay (right) signal strengths for the combination of ATLAS and CMS data [36]. Also shown are the results from each experiment. The error bars indicate the $1 \sigma$ (thick lines) and $2 \sigma$ (thin lines) intervals. The measurements of the global signal strength $\mu$ are also shown.
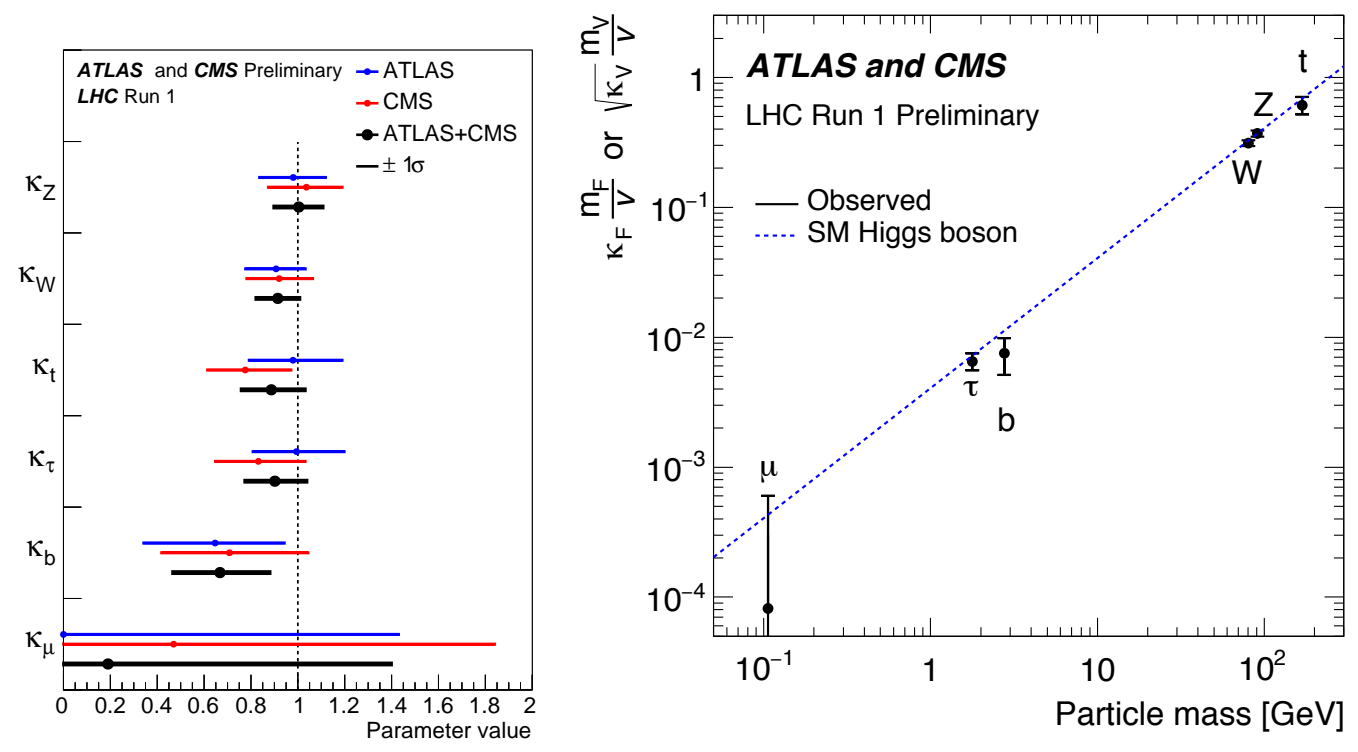

Figure 14: Values of the Higgs coupling modifiers parameters for the combination of ATLAS and CMS data, and separately for each experiment; the error bars indicate the one and two $\sigma$ intervals [36] (left). Values of the coupling modifiers as a function of particle mass; the dashed line indicates the predicted dependence on the particle mass in the case of the SM [36] (right). 

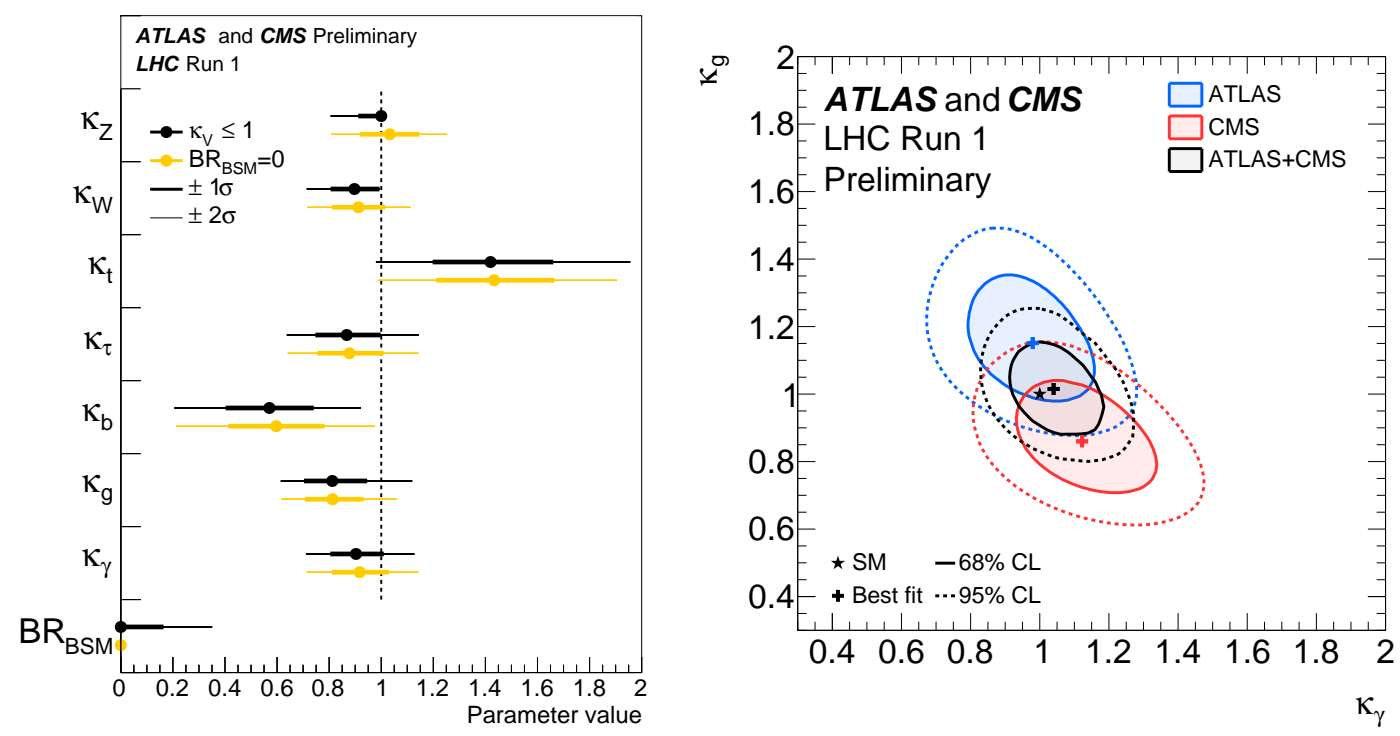

Figure 15: Fit results for the two parameterisations allowing BSM loop couplings [36], with or without additional BSM contributions (BR $\mathrm{BSM}_{\mathrm{BS}}$ ) (left). Negative log-likelihood contours at 68\% and 95\% CL of the photon vs gluon coupling modifiers for the combination of ATLAS and CMS and for each experiment separately [36], constraining all the other coupling modifiers to their SM values and assuming $\mathrm{BR}_{\mathrm{BSM}}=0$ (right).
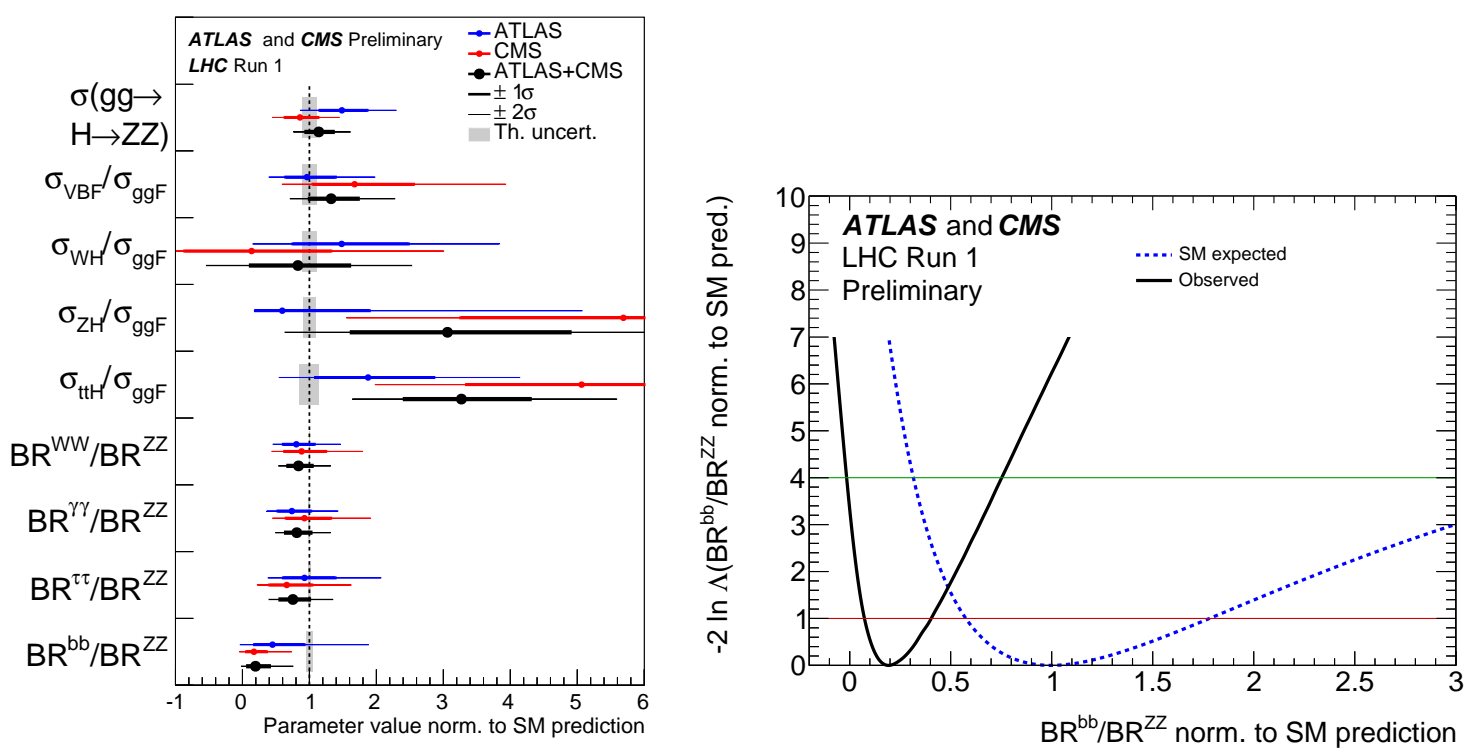

Figure 16: Measurements of the $\mathrm{H} \rightarrow \mathrm{ZZ}$ cross section and of ratios of cross sections and branching ratios, as obtained from the generic parameterisations for ATLAS and CMS, and their combined measurements [36] (left). Observed (solid line) and expected (dashed line) negative log-likelihood scan of the $\mathrm{BR}^{\mathrm{bb}} / \mathrm{BR}^{\mathrm{ZZ}}$ parameter [36] (right). 
tions, showing an observed curve that is 2.5 standard deviations below the SM prediction. As the decay to b-quarks for a Higgs boson with mass $125 \mathrm{GeV}$ is expected to be dominant, around 58\%, the measurement of this coupling, and in general of the Higgs sector coupling to down-type quarks, will be an intriguing challenge with the Run 2 data.

\subsection{First Higgs boson measurements at $13 \mathrm{TeV}$}

Initial measurement of Higgs boson productions with the 2015 proton collision data at $13 \mathrm{TeV}$ have been performed by the ATLAS in the four-lepton [37] and diphoton [38] channels.

Results in the four-lepton channel are shown in Fig. 17. Four signal candidates are expected with SM productions, while $1.0_{-1.5}^{+2.3}$ are fitted, yielding an observed significance of 0.7 standard deviations where 2.8 standard deviations were expected. The fitted inclusive production cross section is $\sigma=12_{-16}^{+25} p b$. Figure 17 also shows limits at the $95 \%$ confidence level (CL) on the production cross section times branching ratio of a heavy scalar boson decaying to four leptons, assuming a narrow intrinsic width for the new particle, with respect to the experimental resolution.
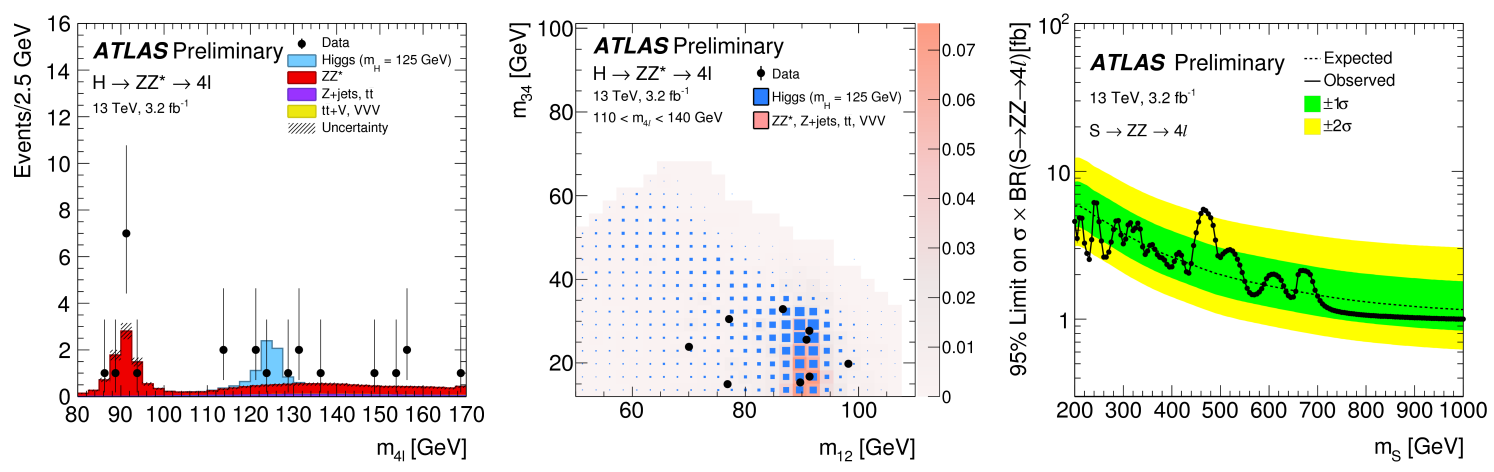

Figure 17: Results in the four-lepton channel [37]. Four-lepton invariant mass distribution of the selected candidates, compared to the background expectation (right). Distributions of data (filled circles) and the expected signal and backgrounds events in the $m_{34} m_{12}$ plane, requiring $m_{4 \ell}$ in the $110-140 \mathrm{GeV}$ range (center). High mass production 95\% CL exclusions limits for $S \rightarrow \mathrm{ZZ} \rightarrow 4 \ell$ in the narrow width approximation (left).

Results in the diphoton channel are shown in Fig. 18. After the inclusive event selection and the fit of the diphoton invariant mass spectrum a total of $113 \pm 74$ (stat $)_{-25}^{+43}$ (syst) signal events are fitted, where 143 events were expected with a SM rate. The observed (expected) significance above the no-signal hypothesis is 1.5 (1.9) standard deviations.

The combination of the diphoton and four-lepton Higgs initial results at $13 \mathrm{TeV}$ by ATLAS yielded an observed signal significance of 1.4 standard deviations, where 3.4 standard deviations were expected with SM production cross-sections. The overall $13 \mathrm{TeV}$ Higgs boson production compatibility with the SM is at the level of 1.3 standard deviations.

\section{Supersymmetry searches}

Supersymmetry (SUSY) introduces a new fundamental symmetry between fermions and bosons. SUSY extensions of the SM have been proposed and searched for over the last 40 years, as attractive and theoretically motivated models offering the possibility of elegant answers and solutions to open problems in the SM and beyond. 

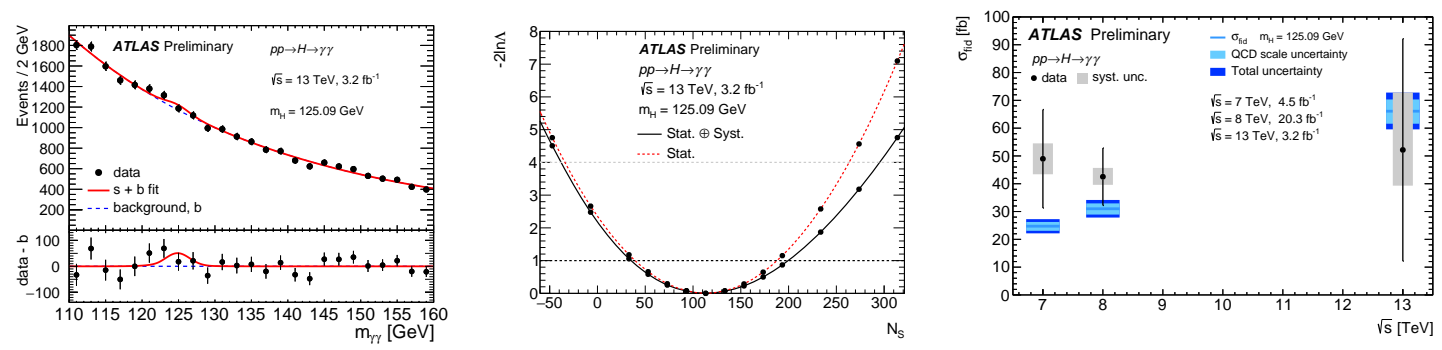

Figure 18: Results in the diphoton channel [38]. ATLAS diphoton invariant-mass spectrum in the 13 $\mathrm{TeV}$ data. The solid red curve shows the fitted signal plus background model with $m_{\mathrm{H}}=125.09 \mathrm{GeV}$, the dotted blue curve shows the fitted background component, and the bottom plot shows the residual of the background-subtracted data (right). The profile likelihood of the number of signal events, with and without systematic uncertainties included (center). ATLAS measured fiducial $\mathrm{H} \rightarrow \gamma \gamma$ cross sections at 7, 8 and 13 $\mathrm{TeV}$ center-of-mass energies (left).

SUSY predicts the existence of additional super partners of all ordinary particles, doubling the SM particle content. SUSY is also a broken in nature, as the masses of ordinary and yet undiscovered SUSY particles (sparticles) appear not to be equal. The main theoretical benefit of SUSY is to ameliorate the fine tuning hierarchy, protecting the Higgs boson mass running from quadratically divergent quantum corrections. SUSY also provides the unification of gauge couplings at the GUT scale $\left(\sim 10^{16} \mathrm{GeV}\right)$, and, in the context of R-parity conserving scenarios, a dark matter candidate in the guise of the Lightest Stable SUSY Particle (LSP).

The search for SUSY with the first 2015 proton collision data at $13 \mathrm{TeV}$ was particularly focused for high mass, strongly produced SUSY gluino and squark pairs as the predicted production cross section enhancement factors with respect to Run 1 were $\sigma(13 \mathrm{TeV}) / \sigma(8 \mathrm{TeV})=15-35$ for sparticles with $M=1-1.5 \mathrm{TeV}$.

\subsection{Jets and missing energy}

ATLAS reported first results of SUSY searches in the jets plus missing energy topology separately for events with up to six jets [39] and above six jets [40].

Figure 19 shows the results for the lower jets multiplicity search [39] where the 'effective mass' $m_{\mathrm{eff}}$ is defined as the scalar sum of the transverse momenta of jets and the missing transverse energy. Good agreement is seen between the observed events in the data and expected from SM background processes, and exclusions are derived in simplified models, that extend the previous reach of Run 1 searches.

Figure 20 shows the results for the high jets multiplicity search [40] where the $E_{\mathrm{T}}^{\mathrm{miss}} / \sqrt{H_{\mathrm{T}}}$ variable is used. Again no excess above Standard Model expectations is observed and exclusions that extending previous limits are derived.

CMS has performed first results of SUSY searches in the jets plus missing energy topology with four distinct methods: (i) with standard jet observables [41], (ii) with the "strasverse mass" $\left(M_{\mathrm{T} 2}\right)$ variable [42], (iii) using the "razor" variables [43] and (iv) with the $\alpha_{\mathrm{T}}$ variable [44].

Figure 21 shows some of the CMS results with searches for SUSY in jets plus missing energy events. Consistently no excess above the expected SM background is observed in all four analysis and exclusion limits are derived in a variety of SUSY simplified models shown in Figure 22. 

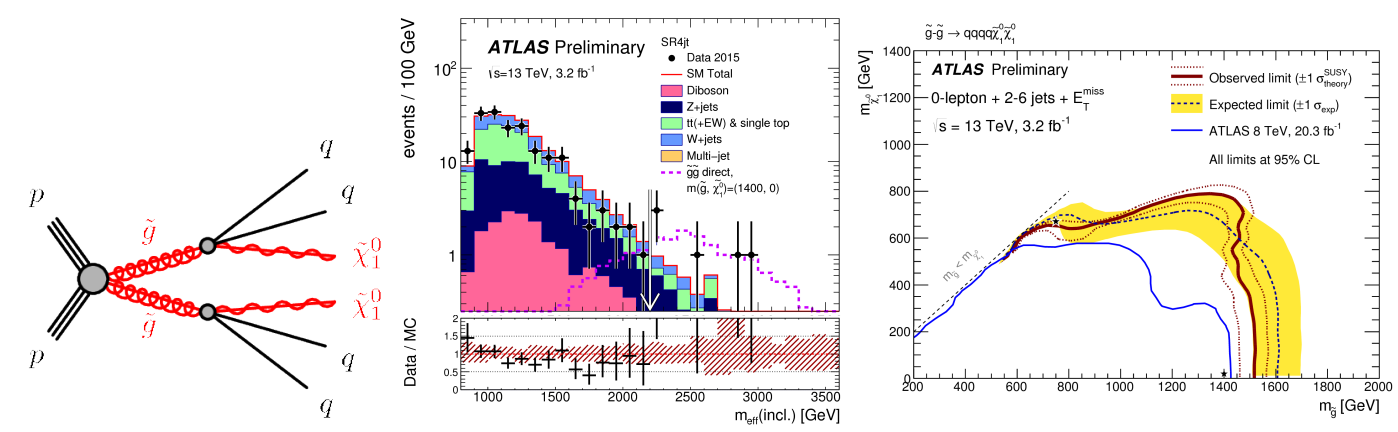

Figure 19: The decay topologies of a gluino-pair production, in simplified models with direct decays of gluinos (left). The ATLAS [39] observed inclusive $m_{\text {eff }}$ distributions for the a signal region with four jets, with MC background and signal benchmark model expectations (center). Exclusion limits for direct production of gluino pairs with decoupled squarks, compared with the observed limits obtained by Run 1 ATLAS searches, in the plane of the LSP mass versus the gluino mass (right).
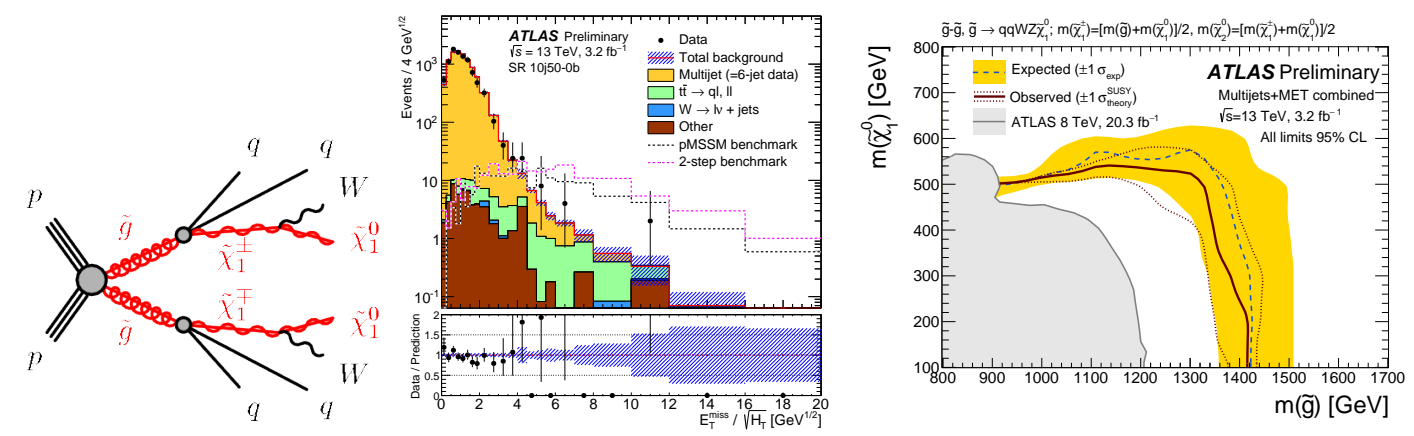

Figure 20: Multijet plus missing energy production in gluino pair decays (left). Distributions of the selection variable $E_{\mathrm{T}}^{\text {miss }} / \sqrt{H_{\mathrm{T}}}$ for the signal region with high jet multiplicity and no b-jet requirement [40] (center). Exclusion curves in the $m_{\tilde{g}}-m_{\text {LSP }}$ plane for a cascade decay model, compared with the observed limits obtained by Run 1 ATLAS searches (right).
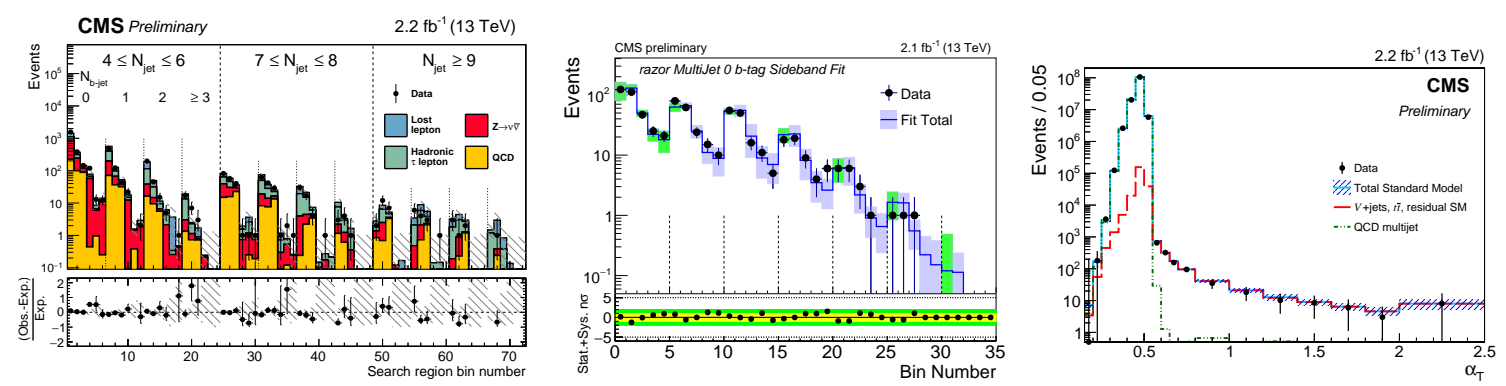

Figure 21: CMS observed numbers of events and corresponding SM background predictions in 72 CMS search regions [41] (left). Comparison of the predicted background with the observed data in bins of $M_{R}$ and $R^{2}$ for the "razor" search [43] (center). The $\alpha_{\mathrm{T}}$ distribution observed in data and compared to expected backgrounds from simulation [44] (right). 

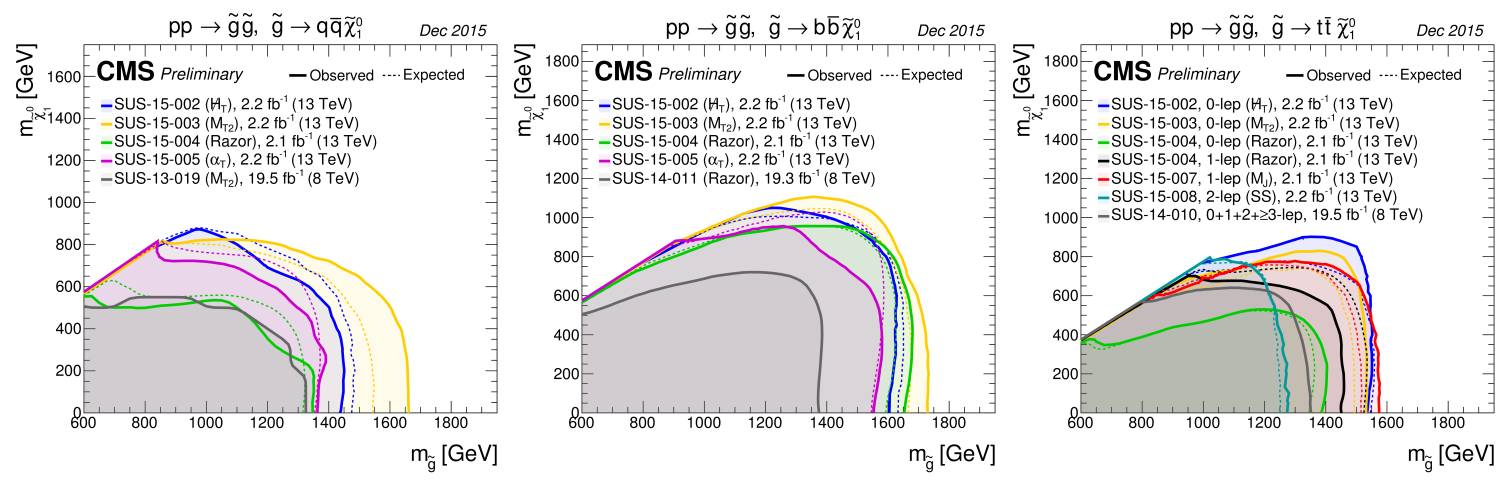

Figure 22: CMS exclusion regions in the plane mass of the lightest neutralino (LSP) versus mass of the gluino with different scenarios for the gluino decay to light quarks (left), bottom quarks (center), or top quarks (right). The exclusion reach of different searches [41, 42, 44, 43] is shown and compared with previous Run 1 results

A search for direct sbottom pair production has been performed by ATLAS in the topology with two b-jets with missing transverse energy using the contransverse mass $\left(m_{\mathrm{CT}}\right)$ [45], as well as a gluino-mediated stop and sbottom pair production search in events with b-jets and missing transverse energy [46]. Some results from these searches are shown in Fig. 23, where data is consistent with the expectations of the SM backgrounds. Derived limits exclude sbottom masses in the 500-800 GeV range, gluino masses in the 1.6-1.8 TeV range, and improve on Run 1 results
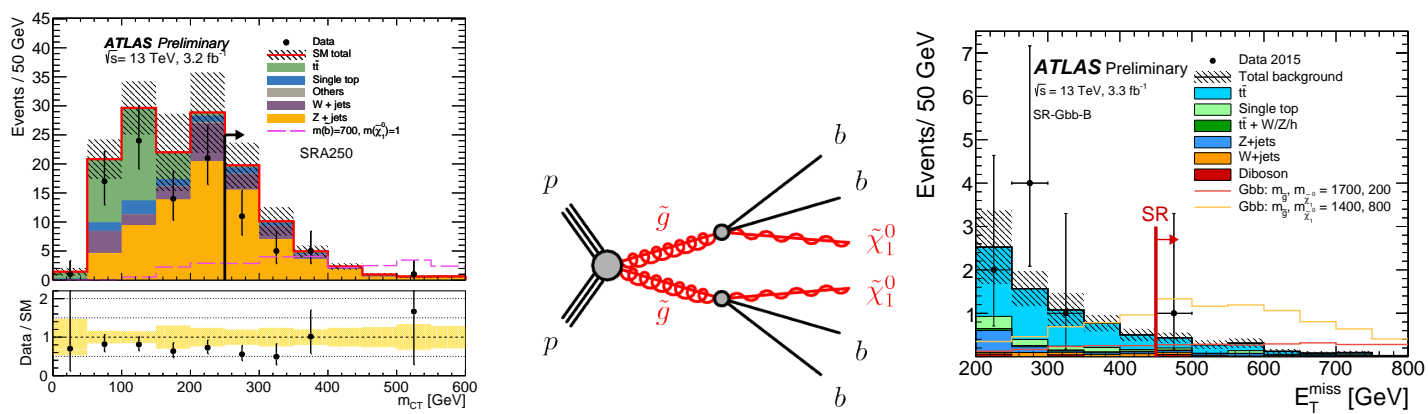

Figure 23: ATLAS distribution of $m_{\mathrm{CT}}$ in a signal region of the direct sbottom pair production search [45] (left). Gluino pair decays to four b-quarks and two LSP (center). Distribution of $E_{\mathrm{T}}^{\text {miss }}$ in a signal region of the multi-b ATLAS SUSY search [46], with example signal models overlaid (right).

ATLAS also performed a search for supersymmetric particles in final states containing a leptonic decay of a $\mathrm{Z}$ boson, jets, and large missing transverse energy [47].

Results for this search are shown in Fig. 24. A total of 21 events are observed on $10.3 \pm 2.3$ expected from background, resulting in an excess of events above the SM expectation, with a significance of 2.2 standard deviations. The analysis updates a search performed with Run 1 data, where of 3.0 standard deviations above the SM predicted yield was found [48].

\subsection{Leptons and jets}

Events with one or two isolated lepton, jets and missing transverse energy are also a promising 

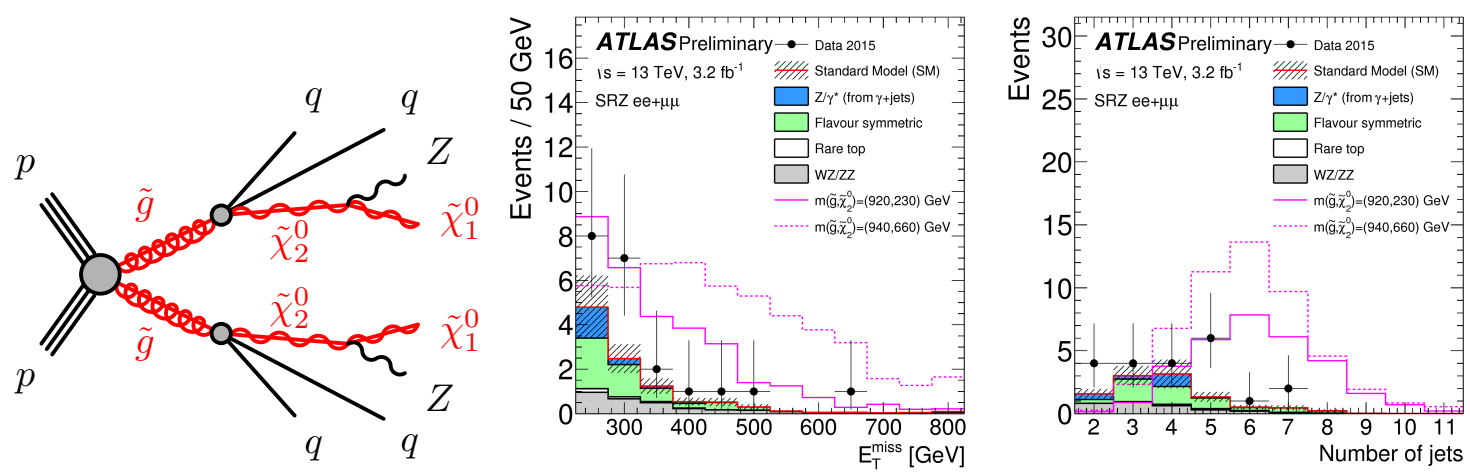

Figure 24: Decay topology involving gluino pair production, with the gluinos following an effective threebody decay to $\mathrm{qq} \chi_{2}^{0}$, and the heavy neutralino decaying as $\chi_{2}^{0} \rightarrow \chi_{1}^{0} \mathrm{Z}$ (left). The missing transverse energy (center) and jet multiplicity (right) for the signal region of the ATLAS SUSY search in the Z boson, jets and missing transverse energy topology [47].

topology to find the presence of strongly produced SUSY signals, from the decay of either gluinos to top quarks [49], or of scalar top pairs produced directly [50]. The searches in $13 \mathrm{TeV}$ pp collision data performed by ATLAS and CMS revealed no excess above SM background expectations, and exclusion limits at 95\% CL were derived for SUSY different models, with a sensitivity similar to the Run 1 exclusions.

\subsection{Same sign leptons}

Strongly produced squark or gluino pairs can give rise to peculiar final states with jets, missing energy and with same-sign leptons that are of particular interest due to lower background levels. Such final states have been promptly investigated by both CMS [51] and ATLAS [52] with the initial $201513 \mathrm{TeV}$ pp collision data, and some of the results are shown in Fig 25.
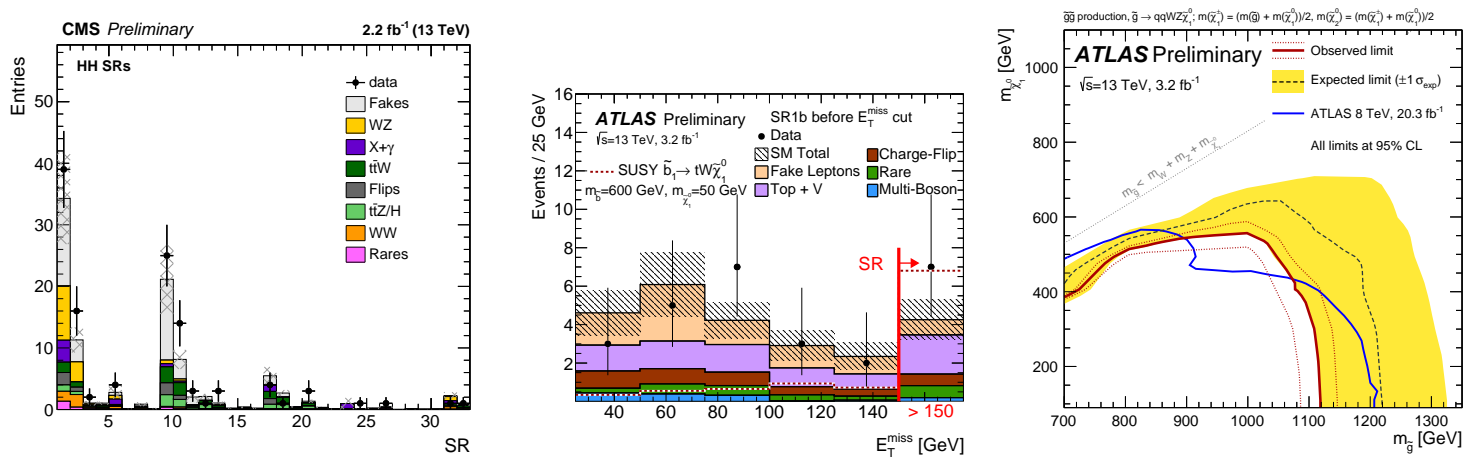

Figure 25: CMS event yields in the "high-high" signal regions [51] (left). ATLAS distribution of $E_{\mathrm{T}}^{\text {miss }}$ in the one b-jet signal region (center), and observed and expected exclusion limits on the gluino, lightest bottom squark and lightest neutralino masses in the context of simplified SUSY scenarios [52], also compared with Run 1 results (right).

The CMS analysis considers multiple search regions defined by missing transverse energy, hadronic transverse energy, transverse mass, number of jets and b-jets, and transverse momenta of 
the leptons in the event. Similar discriminating observables and categorisations are used in the ATLAS search. Dedicated validation regions are defined to test the estimate of the rare SM processes contributing to the signal regions, Here as well, no significant excess over the SM expectations is observed, and the results are interpreted on several simplified SUSY models marginally extending the exclusion limits from previous Run 1 searches.

\section{Conclusions}

A selected and brief summary of CMS and ATLAS results with proton collision from the full Run 1 dataset and from the 2015 Run 2 dataset was given on the topics of electroweak measurements, the Higgs sector, and supersymmetry searches. The combination of CMS and ATLAS Higgs boson Run 1 data provided a highly precise $(0.2 \%)$ mass determination, but quite poor constraints on the Higgs couplings. Initial results with 2015 Run 2 data provide a first glimpse of the precision that can be reached in the study of the electroweak gauge sector and of its symmetry breaking Higgs sector, with the full Run 2 dataset. The 2015 Run 2 data also already extends beyond the Run 1 data in the reach of searches for strongly produced supersymmetric signals, as both CMS and ATLAS collaborations look forward to collect more high energy data, improve the precision measurements, and make new discoveries.

\section{References}

[1] The CMS Collaboration, "The CMS experiment at the CERN LHC," JINST 3 (2008) S08004. doi:10.1088/1748-0221/3/08/S08004

[2] The ATLAS Collaboration, "The ATLAS Experiment at the CERN Large Hadron Collider," JINST 3 (2008) S08003. doi:10.1088/1748-0221/3/08/S08003

[3] S. Bethke, "Results from ATLAS and CMS: Strong Interactions and New Physics", these proceedings.

[4] The CMS Collaboration, "Particle-Flow Event Reconstruction in CMS and Performance for Jets, Taus, and MET," CMS-PAS-PFT-09-001.

[5] The CMS Collaboration, "Commissioning of the Particle-flow Event Reconstruction with the first LHC collisions recorded in the CMS detector," CMS-PAS-PFT-10-001.

[6] The CMS Collaboration, "Commissioning of the Particle-Flow reconstruction in Minimum-Bias and Jet Events from pp Collisions at $7 \mathrm{TeV}$," CMS-PAS-PFT-10-002.

[7] The CMS Collaboration, "Electron and Photon performance using data collected by CMS at $\sqrt{s}=13$ TeV and 25ns", CMS-DP-2015-067.

[8] The CMS Collaboration, "Dimuon spectrum 2015", CMS-DP-2015-055.

[9] M. Cacciari and G. P. Salam, "Dispelling the $N^{3}$ myth for the $k_{t}$ jet-finder," Phys. Lett. B 641 (2006) 57 doi:10.1016/j.physletb.2006.08.037 [hep-ph/0512210].

[10] M. Cacciari, G. P. Salam and G. Soyez, “The Anti-k(t) jet clustering algorithm,” JHEP 0804 (2008) 063 doi:10.1088/1126-6708/2008/04/063 [arXiv:0802.1189 [hep-ph]].

[11] The CMS Collaboration, "Determination of Jet Energy Calibration and Transverse Momentum Resolution in CMS," JINST 6 (2011) P11002 doi:10.1088/1748-0221/6/11/P11002 [arXiv:1107.4277 [physics.ins-det]]. 
[12] The CMS Collaboration, "Pileup Jet Identification,” CMS-PAS-JME-13-005.

[13] The CMS Collaboration, "Identification of b-quark jets with the CMS experiment," JINST 8 (2013) P04013 doi:10.1088/1748-0221/8/04/P04013 [arXiv:1211.4462 [hep-ex]].

[14] The CMS Collaboration, "Identification of b quark jets at the CMS Experiment in the LHC Run 2," CMS-PAS-BTV-15-001.

[15] The CMS Collaboration, "Jet energy scale and resolution performances with 13TeV data", CMS-DP-2016-020.

[16] The CMS Collaboration,"Performance of b-Tagging Algorithms in 25ns Data at 13TeV", CMS-DP-2015-056.

[17] The CMS Collaboration, "Measurement of inclusive $\mathrm{W}$ and $\mathrm{Z}$ boson production cross sections in pp collisions at sqrt(s)=13 TeV," CMS-PAS-SMP-15-004.

[18] The ATLAS collaboration, "Measurement of W and Z Boson Production Cross Sections in pp Collisions at root $\mathrm{s}=13 \mathrm{TeV}$ in the ATLAS Detector," ATLAS-CONF-2015-039.

[19] The CMS Collaboration, "Measurement of the $\mathrm{W}^{+} \mathrm{W}^{-}$cross section in pp collisions at $\sqrt{s}=8 \mathrm{TeV}$ and limits on anomalous gauge couplings," Eur. Phys. J. C 76 (2016) no.7, 401 doi:10.1140/epjc/s10052-016-4219-1 [arXiv:1507.03268 [hep-ex]].

[20] The ATLAS Collaboration, "Measurement of $W^{+} W^{-}$production in pp collisions at $\sqrt{s}=7 \mathrm{TeV}$ with the ATLAS detector and limits on anomalous WWZ and WW $\gamma$ couplings," Phys. Rev. D 87 (2013) no.11, 112001 Erratum: [Phys. Rev. D 88 (2013) no.7, 079906] doi:10.1103/PhysRevD.87.112001, 10.1103/PhysRevD.88.079906 [arXiv:1210.2979 [hep-ex]].

[21] The CMS Collaboration, "Measurement of the WZ production cross section in pp collisions at $\sqrt{s}=13$ TeV," CMS-PAS-SMP-15-006.

[22] The ATLAS Collaboration, "Measurement of the ZZ Production Cross Section in pp Collisions at $\sqrt{s}$ $=13$ TeV with the ATLAS Detector," Phys. Rev. Lett. 116 (2016) no.10, 101801 doi:10.1103/PhysRevLett.116.101801 [arXiv:1512.05314 [hep-ex]].

[23] The CMS Collaboration, "Measurement of the ZZ production cross section in $\ell \ell \ell^{\prime} \ell$ ' decays in pp collisions at $\sqrt{s}=13$ TeV," CMS-PAS-SMP-15-005.

[24] A. Salam and J. C. Ward, “On A Gauge Theory Of Elementary Interactions,” Nuovo Cim. 19 (1961) 165. doi:10.1007/BF02812723

[25] S. L. Glashow, “Partial Symmetries of Weak Interactions,” Nucl. Phys. 22 (1961) 579. doi:10.1016/0029-5582(61)90469-2

[26] S. Weinberg, “A Model of Leptons,” Phys. Rev. Lett. 19 (1967) 1264. doi:10.1103/PhysRevLett.19.1264

[27] F. Englert and R. Brout, "Broken Symmetry and the Mass of Gauge Vector Mesons," Phys. Rev. Lett. 13 (1964) 321. doi:10.1103/PhysRevLett.13.321

[28] P. W. Higgs, “Broken Symmetries and the Masses of Gauge Bosons,” Phys. Rev. Lett. 13 (1964) 508. doi:10.1103/PhysRevLett.13.508

[29] G. S. Guralnik, C. R. Hagen and T. W. B. Kibble, "Global Conservation Laws and Massless Particles,” Phys. Rev. Lett. 13 (1964) 585. doi:10.1103/PhysRevLett.13.585 
[30] The ALEPH and DELPHI and L3 and OPAL and LEP Electroweak Collaborations, "Electroweak Measurements in Electron-Positron Collisions at W-Boson-Pair Energies at LEP,” Phys. Rept. 532 (2013) 119 doi:10.1016/j.physrep.2013.07.004 [arXiv:1302.3415 [hep-ex]].

[31] The CMS Collaboration, "Observation of a new boson at a mass of $125 \mathrm{GeV}$ with the CMS experiment at the LHC,” Phys. Lett. B 716 (2012) 30 doi:10.1016/j.physletb.2012.08.021 [arXiv:1207.7235 [hep-ex]].

[32] The ATLAS Collaboration, "Observation of a new particle in the search for the Standard Model Higgs boson with the ATLAS detector at the LHC," Phys. Lett. B 716 (2012) 1 doi:10.1016/j.physletb.2012.08.020 [arXiv:1207.7214 [hep-ex]].

[33] The ATLAS and CMS Collaborations, "Combined Measurement of the Higgs Boson Mass in $p p$ Collisions at $\sqrt{s}=7$ and $8 \mathrm{TeV}$ with the ATLAS and CMS Experiments," Phys. Rev. Lett. 114 (2015) 191803 doi:10.1103/PhysRevLett.114.191803 [arXiv:1503.07589 [hep-ex]].

[34] The ATLAS Collaboration, "Measurement of the Higgs boson mass from the $H \rightarrow \gamma \gamma$ and $H \rightarrow Z Z^{*} \rightarrow 4 \ell$ channels with the ATLAS detector using $25 \mathrm{fb}^{-1}$ of $p p$ collision data," Phys. Rev. D 90 (2014) no.5, 052004 doi:10.1103/PhysRevD.90.052004 [arXiv:1406.3827 [hep-ex]].

[35] The CMS Collaboration, "Observation of the diphoton decay of the Higgs boson and measurement of its properties,” Eur. Phys. J. C 74 (2014) no.10, 3076 doi:10.1140/epjc/s10052-014-3076-Z [arXiv:1407.0558 [hep-ex]]

[36] The ATLAS and CMS Collaborations, "Measurements of the Higgs boson production and decay rates and constraints on its couplings from a combined ATLAS and CMS analysis of the LHC pp collision data at $\sqrt{s}=7$ and 8 TeV," arXiv:1606.02266 [hep-ex].

[37] The ATLAS collaboration, "Measurements of the Higgs boson production cross section at 7, 8 and 13 $\mathrm{TeV}$ centre-of-mass energies and search for new physics at $13 \mathrm{TeV}$ in the $H \rightarrow Z Z^{*} \rightarrow \ell^{+} \ell \ell^{\prime+} \ell^{\prime}$ final state with the ATLAS detector", ATLAS-CONF-2015-059.

[38] The ATLAS collaboration, "Measurement of the Higgs boson production cross section at 7, 8 and 13 $\mathrm{TeV}$ center-of-mass energies in the $H \rightarrow \gamma \gamma$ channel with the ATLAS detector", ATLAS-CONF-2015-060.

[39] The ATLAS collaboration, "Search for squarks and gluinos in final states with jets and missing transverse momentum at $\sqrt{s}=13 \mathrm{TeV}$ with the ATLAS detector," ATLAS-CONF-2015-062.

[40] The ATLAS collaboration, "Search for new phenomena in final states with large jet multiplicities and missing transverse momentum with ATLAS using $\sqrt{s}=13 \mathrm{TeV}$ protonâĂŞproton collisions," ATLAS-CONF-2015-077.

[41] The CMS Collaboration, "Search for supersymmetry in the multijet and missing transverse momentum channel in pp collisions at 13 TeV," CMS-PAS-SUS-15-002.

[42] The CMS Collaboration, "Search for new physics in the all-hadronic final state with the MT2 variable," CMS-PAS-SUS-15-003.

[43] The CMS Collaboration, "Inclusive search for supersymmetry using the razor variables at sqrt(s)=13 TeV," CMS-PAS-SUS-15-004.

[44] The CMS Collaboration, "Search for new physics in final states with jets and missing transverse momentum in $\sqrt{s}=13 \mathrm{TeV}$ pp collisions with the $\alpha_{\mathrm{T}}$ variable," CMS-PAS-SUS-15-005.

[45] The ATLAS collaboration, "Search for Bottom Squark Pair Production with the ATLAS Detector in proton-proton Collisions at $\sqrt{s}=13 \mathrm{TeV}$," ATLAS-CONF-2015-066. 
[46] The ATLAS collaboration, "Search for pair-production of gluinos decaying via stop and sbottom in events with $b$-jets and large missing transverse momentum in $\sqrt{s}=13 \mathrm{TeV} p p$ collisions with the ATLAS detector," ATLAS-CONF-2015-067.

[47] The ATLAS collaboration, "A search for Supersymmetry in events containing a leptonically decaying $Z$ boson, jets and missing transverse momentum in $\sqrt{s}=13 \mathrm{TeV} p p$ collisions with the ATLAS detector," ATLAS-CONF-2015-082.

[48] The ATLAS Collaboration, "Search for supersymmetry in events containing a same-flavour opposite-sign dilepton pair, jets, and large missing transverse momentum in $\sqrt{s}=8 \mathrm{TeV}$ pp collisions with the ATLAS detector,' Eur. Phys. J. C 75 (2015) no.7, 318 Erratum: [Eur. Phys. J. C 75 (2015) no.10, 463] doi:10.1140/epjc/s10052-015-3661-9, 10.1140/epjc/s10052-015-3518-2 [arXiv:1503.03290 [hep-ex]].

[49] The CMS Collaboration, "Search for supersymmetry in pp collisions at sqrt (s) = $13 \mathrm{TeV}$ in the single-lepton final state using the sum of masses of large radius jets," CMS-PAS-SUS-15-007.

[50] The ATLAS collaboration, "Search for gluinos in events with an isolated lepton, jets and missing transverse momentum at ấĽ s= 13 TeV with the ATLAS detector," ATLAS-CONF-2015-076.

[51] The CMS Collaboration, "Search for SUSY in same-sign dilepton events at sqrts=13 TeV," CMS-PAS-SUS-15-008.

[52] The ATLAS collaboration, "Search for supersymmetry at $\sqrt{s}=13 \mathrm{TeV}$ in final states with jets and two same-sign leptons or three leptons with the ATLAS detector," ATLAS-CONF-2015-078. 